\title{
MicroRNAs and zinc metabolism-related gene expression in prostate cancer cell lines treated with zinc(II) ions
}

\author{
MARIAN HLAVNA ${ }^{1,4}$, MARTINA RAUDENSKA ${ }^{1,2}$, KRISTYNA HUDCOVA $^{1}$, JAROMIR GUMULEC $^{1,3}$, \\ MARKETA SZTALMACHOVA ${ }^{1,2}$, VERONIKA TANHÄUSEROVA ${ }^{1}$, PETR BABULA ${ }^{4}$, VOJTECH ADAM ${ }^{2,3}$, \\ TOMAS ECKSCHLAGER ${ }^{5}$, RENE KIZEK ${ }^{2,3}$ and MICHAL MASARIK ${ }^{1,3}$ \\ ${ }^{1}$ Department of Pathological Physiology, Faculty of Medicine, Masaryk University, CZ-625 00 Brno; \\ ${ }^{2}$ Department of Chemistry and Biochemistry, Mendel University in Brno, CZ-613 00 Brno; ${ }^{3}$ Central European Institute \\ of Technology, Brno University of Technology, CZ-616 00 Brno; ${ }^{4}$ Department of Natural Drugs, Faculty of Pharmacy, \\ University of Veterinary and Pharmaceutical Sciences, CZ-612 42 Brno; ${ }^{5}$ Department of Paediatric Haematology \\ and Oncology, Second Faculty of Medicine, Charles University and University \\ Hospital Motol/V Uvalu 84, CZ-150 06 Prague 5, Czech Republic
}

Received July 10, 2012; Accepted September 13, 2012

DOI: 10.3892/ijo.2012.1655

\begin{abstract}
MicroRNAs (miRNAs) are a large class of singlestranded RNA molecules involved in post-transcriptional gene silencing. miRNAs not only regulate various developmental and physiological processes but also are involved in cancer development. Additionally, they can be considered as biomarkers of some pathological processes. The aim of this study was to determine the expression levels of selected miRNA and zinc(II)related genes (ZIP-1, BAX, MT2A and MT1A) in the non-tumor PNT1A prostate cell line in comparison with the prostate cancer cell lines 22Rv1, PC-3 and LNCaP after zinc(II) treatment. Using bioinformatic approaches we selected miRNAs with putative binding sites in the 3'UTR regions in Metallothionein 1A and 2A as miRNA 23a, 141, 224, 296-3p, 320, 375 and 376. We observed significantly higher expression of miRNA 23a in all tumor lines compared to non-tumor PNT1A (13.6-fold in 22Rv1, 7.3-fold in PC-3, 8.3-fold in LNCaP, p<0.01). We also observed that the $22 \mathrm{Rv} 1$ cell line has significantly higher expression of miRNA 224 in comparison to other cell lines. In addition, all tumor cell lines expressed significantly higher levels of miRNA 375 in comparison to non-tumor PNT1A (87.1-fold in 22Rv1, nearly 2,000-fold in PC-3, 56.3-fold in LNCaP, $\mathrm{p}<0.01$ ). Nevertheless, miRNA 375 and 23a expression levels strongly suggest their potential to contribute to the diagnosis of prostate cancer and miRNA 224 eventually may be suitable for classification of primary tumors. The expression of miRNA 224 in $22 \mathrm{Rv} 1$ cell line was negatively correlated with increasing
\end{abstract}

Correspondence to: Dr Michal Masarik, Department of Pathological Physiology, Faculty of Medicine, Masaryk University, Kamenice 5, CZ-625 00 Brno, Czech Republic

E-mail:masarik@med.muni.cz

Key words: miRNA, prostate cancer, polymerase chain reaction, metallothionein, zinc(II) ions
zinc(II) concentration only. Our experiments revealed significant negative correlation of miRNA 376 and $M T 2 A$ in $22 \mathrm{Rv} 1$ and a negative correlation between miRNA 224 and MT1A in PC-3 cells which may denote possible direct regulation of $M T$ genes by specific miRNAs in prostate cancer.

\section{Introduction}

Prostate cancer is the most frequently diagnosed cancer in men in Western countries (1). The therapeutic success of prostate cancer treatment can be enormously improved if the disease is diagnosed soon. Thus, the search for suitable biomarkers used for early detection of the presence and progression of the disease, as well as the prediction after the clinical interventions is ongoing. The current prostate cancer biomarkers do not fulfill all a forementioned requirement as there remains a lack of reliable biomarkers that can specifically distinguish between those patients who should be treated adequately to stop the aggressive form of the disease and those who should avoid overtreatment of the indolent form (2). Specific microRNAs (miRNAs) have been shown to serve key functional roles in prostate cancer (PC) cells, as discovered mainly by overexpression and knockdown experiments in cell cultures (3-5). Key cellular processes such as proliferation, apoptosis, survival, invasion, migration, cell cycle progression and androgen signaling, are regulated by miRNAs in PC cells (6-8). From biochemical point of view, miRNAs are small, phylogenetically conserved, noncoding RNAs that are able to regulate expression of at least $30 \%$ of all protein encoding genes (9). Mature miRNAs are involved in post-transcriptional gene silencing. They arise from intergenic or intragenic (both exonic and intronic) genomic regions that are transcribed as long primary transcripts. Recently, miRNAs have received attention as potential cancer diagnostic markers and therapeutic targets (10). miRNA expression profiles have been shown to classify different cancers and identify cancer tissue origins (11). Deregulated miRNA expression was reported in various human cancers including prostate cancer (12). Of particular note is 
the polycistronic set of microRNAs consisting of miRNA 17, miRNA 18, miRNA 19a, miRNA 19b and miRNA 92, which are overexpressed in leukemic lines compared with the other cell lines (11). miRNAs expressions can also predict prostate cancer biochemical relapse and are involved in tumor progression (10). miRNA 375 was considered as useful to identify patients with metastatic disease as well as for a positive lymph node status. miRNA 141 was identified as upregulated in the serum of patients with higher grade prostate tumors (13).

The changes of miRNA synthesis profile can be related to changes in prostate cell metabolism. Among metabolic changes, prostate cancer cells have altered ability to uptake and to accumulate zinc(II) ions compared to healthy prostate tissue (14) and one can suggest that miRNAs could be somewhat connected with this phenomenon. Zinc(II) ions themselves play a role in proliferation, differentiation regulation and apoptosis in prostate $(15,16)$. Extracellular zinc(II) ions can increase the formation of insulin-like growth factor (IGF) (17) and stimulate the epidermal growth factor-receptor (EGF-R) (18). At the level of protein phosphorylation uptake of zinc(II) and/or their releasing from zincosomes can modulate the activity of cyclic nucleotide phosphodiesterase (PDE) (19), mitogen-activated protein kinase (MAPK) (20), protein kinase C (PKC), protein tyrosine phosphatases (PTP), $\mathrm{Ca}$ (II)-calmodulin activated protein kinase-2 (CaMPK-2) and P70S6 kinase (P70S6K) (21). Altogether with the fact that zinc(II) is involved in apoptosis via translocation of existing resident cytosolic Bax to mitochondria and its increasing total cellular Bax level (22), it is highly likely that zinc(II) plays an important role in prostate cancer pathogenesis (23).

In light of above mentioned facts, it can be assumed that altered zinc(II) metabolism will have a great impact on prostate cancer tumorigenesis. Zinc(II) homeostasis and metabolism in healthy and tumor tissues are maintained mainly by two types of proteins: i) zinc-binding proteins [mostly by metallothioneins, a family of low-molecular-mass $(6-10 \mathrm{kDa})$ proteins characterized by a high cysteine content and a high binding capacity for post-transition metal ions (24)], which act as buffer and donor of zinc(II) for intracellular processes and ii) zinc transporters (ZIP and $\mathrm{ZnT}$ protein families), which are responsible for zinc(II) fluxes into/from cells and organelles, because zinc(II) can not freely pass though membranes (25).

In this study we focused on synthesis of miRNAs as miRNA 23a, 141, 224, 296-3p, 320, 375 and 376, which have putative binding sites within 3'UTR of metallothionein genes (MT2A, MT1A) in the non-tumor PNT1A prostate cell line and prostate cancer cell lines 22Rv1, PC-3, and LNCaP treated with zinc(II) ions. Our previous studies have provided the evidence of the association between metallothionein (MT) expression and prostate tumor progression (26). Post-transcriptional gene silencing can be involved in metallothionein regulation and different levels of miRNAs in tumor and non-tumor lines can be expected. Therefore, we also determined expression levels of ZIP-1, BAX, MT2A and MTIA genes in the treated cell lines.

\section{Materials and methods}

Chemical and biochemical reagents. RPMI-1640 medium, Ham's F12 medium, fetal bovine serum (FBS) - mycoplasma free, penicillin/streptomycin and trypsin EDTA were purchased from PAA Laboratories GmbH (Pasching, Austria). PBS was purchased from Invitrogen Corp. (Carlsbad, CA, USA). EDTA, zinc(II) sulfate, RIPA buffer and all other chemicals of ACS purity were purchased from Sigma Aldrich Co. (St. Louis, MO, USA), unless noted otherwise.

miRNA selection. Software Targetscan (http://www.targetscan. org) was used for the selection of suitable miRNAs. miRNAs were selected according to the putative binding sites within 3'UTR of metallothionein genes (MT2A, MT1A).

Cell cultures. Four human prostatic cell lines were used in this study: i) PNT1 A human cell line established by immortalisation of normal adult prostatic epithelial cells by transfection with a plasmid containing SV40 genome with a defective replication origin. The primary culture was obtained from the prostate of a 35-year-old male at post mortem; ii) cell line 22Rv1 has been derived from a primary human prostatic carcinoma xenograft, CWR22R. iii) LNCaP cells are androgen-sensitive human prostate adenocarcinoma cells derived from the left supraclavicular lymph node metastasis from a 50-year-old Caucasian male; iv) PC-3 human cell line established from a grade 4 androgen independent and unresponsive prostatic adenocarcinoma from 62-year-old Caucasian male and derived from metastatic site in bone. All cell lines used in this study were purchased from Health Protection Agency Culture Collections (Salisbury, UK).

Culture conditions. PNT1A, LNCaP and 22Rv1 cells were cultured in RPMI-1640 medium with 10\% FBS. PC-3 cells were cultured in Ham's F12 medium with 7\% FBS. All media were supplemented with penicillin $(100 \mathrm{U} / \mathrm{ml})$ and streptomycin $(0.1 \mathrm{mg} / \mathrm{ml})$, and the cells were maintained at $37^{\circ} \mathrm{C}$ in a humidified incubator (Sanyo, Japan) with $5 \% \mathrm{CO}_{2}$. The sub-cultivations of all cell lines were carried out within the range from 10 to $35 \mathrm{~h}$.

Zinc(II) treatment. Cells were treated with five concentrations of $\mathrm{ZnSO}_{4}(0-90 \mu \mathrm{mol} / \mathrm{l})$ at $37^{\circ} \mathrm{C}$ for $48 \mathrm{~h}$ after cells reached $\sim 50 \%$ confluence. Cells were then harvested and washed four times with 1X PBS, pH 7.4.

RNA and miRNA isolation and reverse transcription. High pure total-RNA isolation kit (Roche, Basel, Switzerland) was used for RNA isolation and high pure miRNA isolation kit for miRNA isolation. For both isolations the medium was removed and samples were twice washed with $5 \mathrm{ml}$ of ice-cold PBS. Cells were scraped off, transferred to clean tubes and centrifuged at $20,800 \mathrm{xg}$ for $5 \mathrm{~min}$ at $4^{\circ} \mathrm{C}$. After this step, lysis buffer was added and RNA isolation was carried out according to manufacturer's instructions. Isolated RNA and miRNA was used for cDNA synthesis. RNA (600 ng) was transcribed using Transcriptor first strand cDNA synthesis kit (Roche, Switzerland) and $10 \mathrm{ng}$ of miRNA was transcribed using TaqMan ${ }^{\circledR}$ microRNA reverse transcription kit (Applied Biosystems, Carlsbad, CA, USA) according to manufacturer's instructions. Prepared cDNA (20 $\mu$ l) from total-RNA was diluted with RNase-free water to $100 \mu \mathrm{l}$ and $5 \mu \mathrm{l}$ was directly analyzed by 7500 real-time PCR system (Applied Biosystems). In case of miRNA analysis $1.33 \mu \mathrm{l}$ of transcribed miRNA was used directly in real-time PCR reaction according to manufacturer's instruction. 


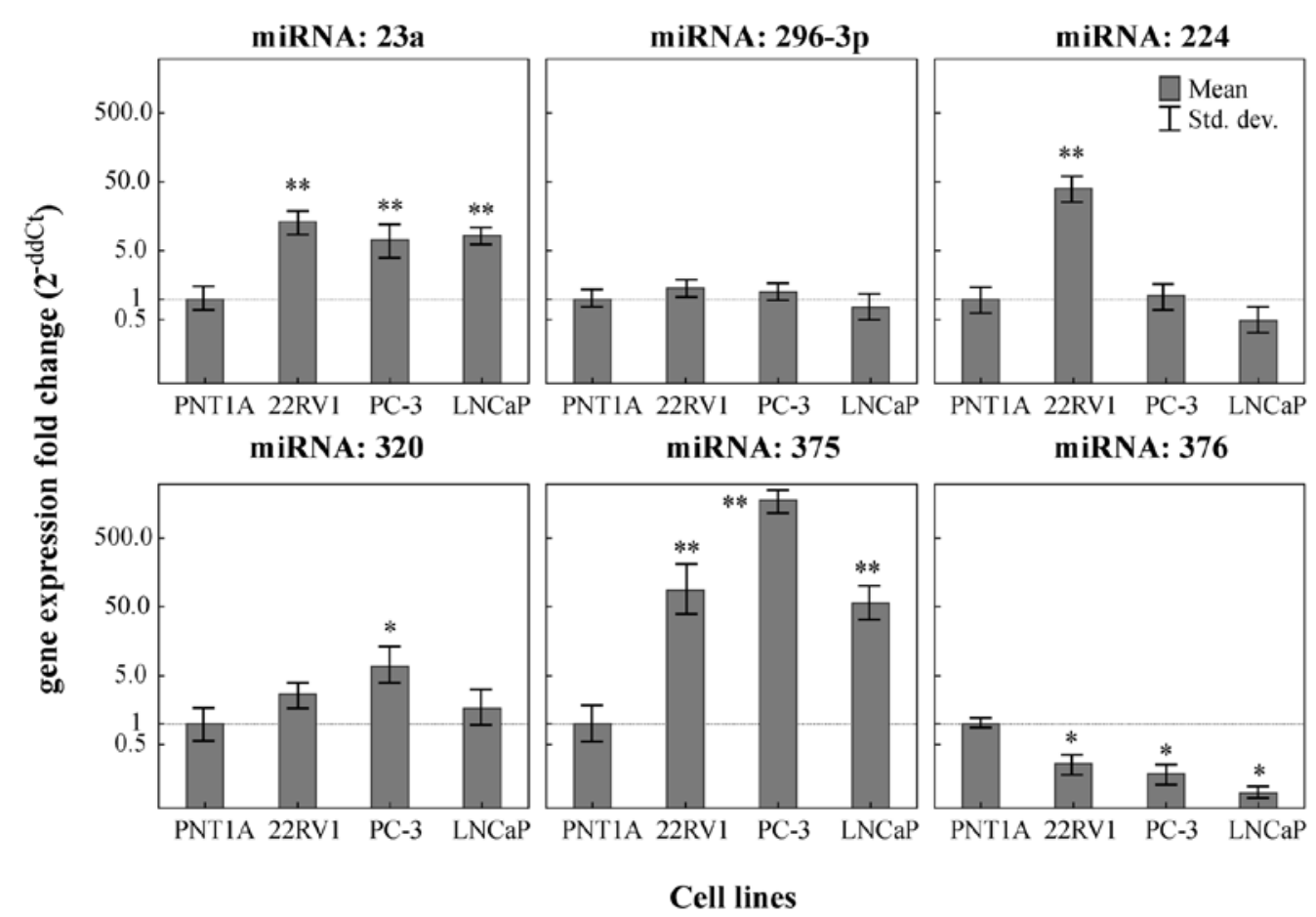

Figure 1. miRNA expression levels without zinc(II) treatment in 22Rv1, PC-3 and LNCaP cell lines relative to non-tumor PNT1A cell line. Base line transcription level of selected miRNAs was conducted by RT-PCR. For other experimental parameters see Materials and methods. " p $<0.05 ;{ }^{* * *} \mathrm{p}<0.01$.

Quantitative polymerase chain reaction $(q-P C R)$. Q-PCR was performed in triplicate using the TaqMan gene expression assay system with the 7500 real-time PCR system (Applied Biosystems) and the amplified DNA was analyzed by the comparative $\mathrm{Ct}$ method using $\beta$-actin as an endogenous control for metallothionein MT1A and MT2A, Bax and ZIP-1 gene expression quantification. The primer and probe sets for $\beta$-actin (assay ID: $\left.H s 99999903 \_m l\right), M T 1 A$ (assay ID: Hs00831826_ s1), MT2A (Hs02379661_gl) ZIP-1 (Hs00205358_m1) and Bax (Hs00180269_m1) were selected from TaqMan gene expression assays. Primer probe sets for quantification hsa-miRNA-23a (assay ID: 000399), hsa-miR-141 (assay ID: 000463), hsa-miR-224 (assay ID: 002099), hsa-miR-296 (assay ID: 000527), hsa-miR-320 (assay ID: 002277), hsa-miR-375 (assay ID: 000564), hsa-miR-376 (assay ID: 000565) were selected from TaqMan miRNA expression assays. Q-PCR was performed under the following amplification conditions: total volume of $20 \mu \mathrm{l}$, initial incubation $50^{\circ} \mathrm{C} / 2 \mathrm{~min}$ followed by denaturation $95^{\circ} \mathrm{C} / 10 \mathrm{~min}$, then 45 cycles $95^{\circ} \mathrm{C} / 15 \mathrm{sec}, 60^{\circ} \mathrm{C} / 1 \mathrm{~min}$.

Statistics. The data were analyzed using StatSoft Statistica version 10 (www.statsoft.com). Mann-Whitney U test was used to reveal differences in the gene expression. Associations within variables were determined using the Pearson correlation. $\mathrm{p}<0.05$ was considered significant.

\section{Results}

miRNA and mRNA baseline expression without zinc(II) treatment. Primarily, we aimed at determination of baseline level of six miRNAs (23a, 296-3p, 224, 320, 375 and 376) selected according to the putative binding sites within 3'UTR of metallo- thionein genes (MT2A, MT1A) and genes MT1A, MT2A, ZIP-1 and $B A X$ in cell lines used in this study as PNT1A (control) and 22Rv1, LNCaP and PC-3 (cancer cell lines). The relative expression of miRNAs and mRNAs in tumor cell lines was expressed as a relative expression fold change compared to the baseline expression of non-tumor PNT1A cell line.

The differences in expression of particular miRNAs are shown in Fig. 1. We observed significantly higher relative expression of miRNA 23a in all tumor lines compared to non-tumor PNT1A (13.6-fold in 22Rv1, 7.3-fold in PC-3, 8.3-fold in LNCaP, $\mathrm{p}<0.01)$. Similarly, all tumor lines expressed miRNA 375 in significantly higher levels in comparison with non-tumor PNT1A (87.1-fold in 22Rv1, 1776.7-fold in PC-3, 56.3-fold in LNCaP, $\mathrm{p}<0.01)$. On the contrary, lower relative expression of miRNA 376 in all tumor lines in comparison with non-tumor PNT1A was observed (3-fold in 22Rv1, 5-fold in PC-3, 10-fold in LNCaP, $p<0.05)$. We also found significantly higher $(\mathrm{p}<0.05)$ relative expression of miRNA 320 in PC-3 line compared to other tumor and non-tumor lines. Significantly higher $(\mathrm{p}<0.01)$ expression of miRNA 224 in 22Rv1 line compared to other lines has been observed and no differences between cell lines were observed in expression of miRNA 296-3p.

The differences in expression of the selected mRNAs among cell lines are shown in Fig. 2. The expression of Bax mRNA is significantly higher in LNCaP cell line (10.5-fold, $\mathrm{p}=0.03)$ in comparison with non-tumor PNT1A. Level of MT1A expression in 22Rv1 cell line is higher with marginal significance (1.8-fold, $\mathrm{p}=0.055)$ in comparison with PNT1A. On the other hand significantly higher MT1A expression is found in $\mathrm{LNCaP}$ and PC-3 cell lines (10-fold, $\mathrm{p}=0.03$ ) with comparison to relative expression in PNT1A. The expression of MT2A was higher in all tumor lines in comparison with PNT1A (2.0-fold, $\mathrm{p}=0.05$ in 


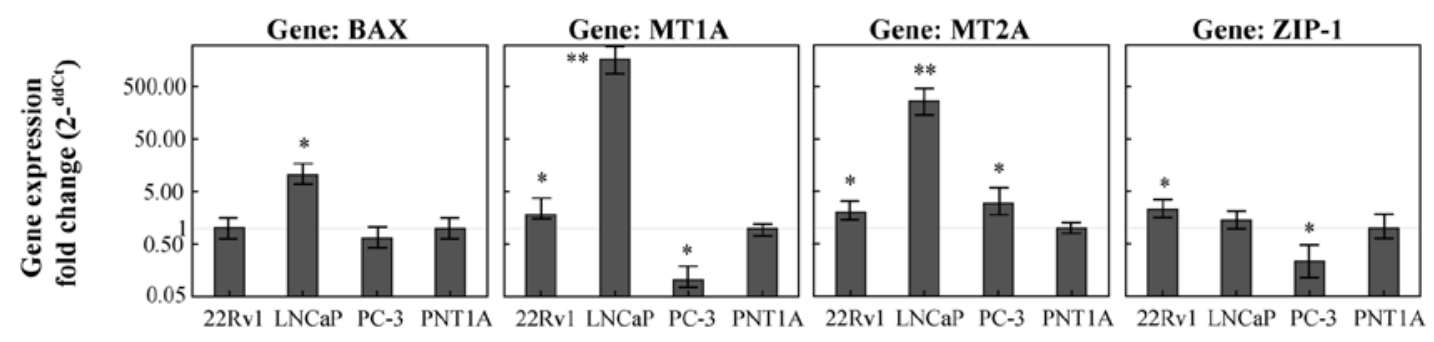

Figure 2. mRNA baseline expression levels of selected genes without zinc(II) treatment in 22Rv1, PC-3 and LNCaP cell lines relative to non-tumor PNT1A cell line. Base line transcription level of selected genes (BAX, MT1A, MT2A and ZIP-1) was conducted by RT-PCR. For other experimental parameters see Materials and methods. ${ }^{*} \mathrm{p}<0.05 ;{ }^{* *} \mathrm{p}<0.002$.

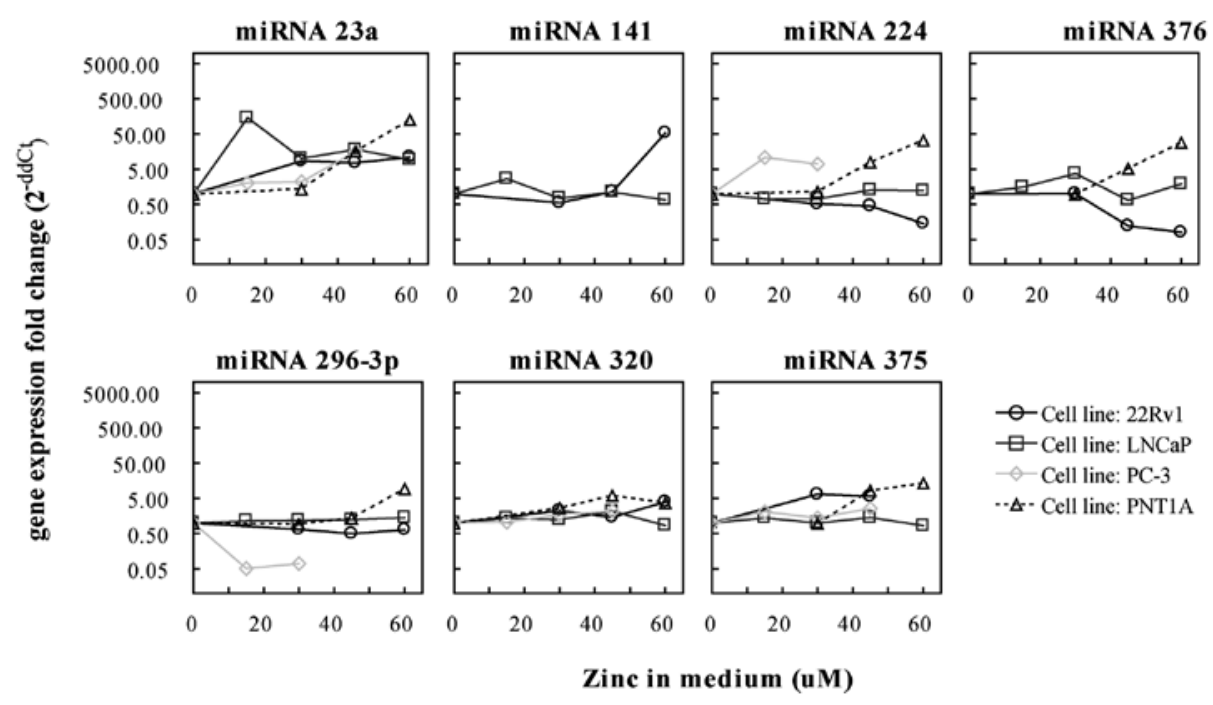

Figure 3. Gene expression fold change of selected miRNAs after zinc(II) treatment. MiRNA 23a shows significant elevating trend after zinc(II) treatment in all cell lines. Other miRNAs demonstrate cell line-specific trends. For other experimental parameters see Materials and methods.

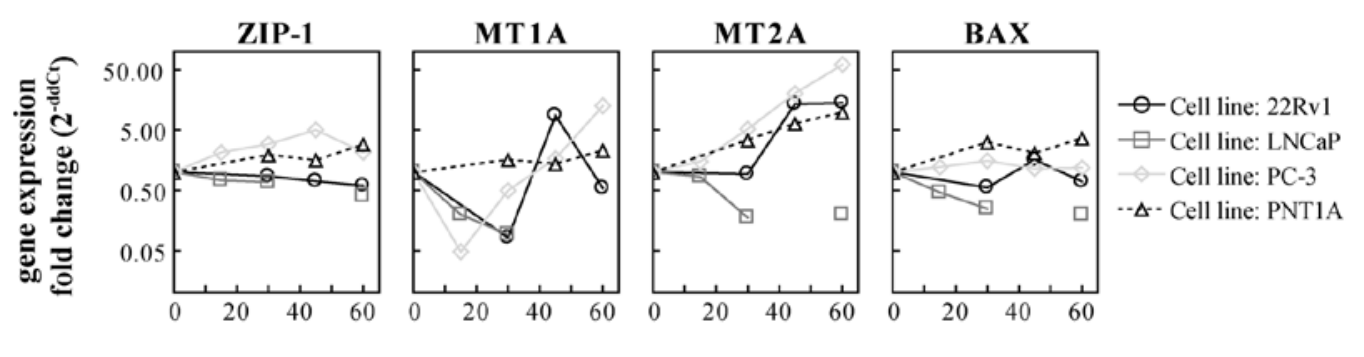

Zinc in medium $(\mu \mathrm{M})$

Figure 4. Dependence between mRNA expression levels and zinc(II) concentration in medium. Level of MT2A expression in PC3 and 22Rv1 cell lines and ZIP-1 in PNT1A cell line were significantly increased by the zinc(II) treatment. Expression of ZIP-1 in LNCaP cell line was significantly decreased by increasing zinc(II) concentration. For other experimental parameters see Materials and methods.

22Rv1, 261.4-fold, $\mathrm{p}=0.002$ in LNCaP, and 2.9-fold, $\mathrm{p}=0.05$ in PC-3 cell line). Moreover, we observed higher relative expression of ZIP-1 in 22Rv1 (2.3-fold, p=0.05) and lower relative expression of ZIP-1 in PC-3 cell line compared to non-tumor PNT1A (5-fold, $\mathrm{p}=0.05$ ).

miRNA and mRNA expression after zinc(II) treatment. In addition, we determined expression levels of selected miRNA after zinc(II) treatment. Trends in miRNA expressions under zinc(II) treatment are shown in Fig. 3. MiRNA 23a shows significant elevating trend after zinc(II) treatment in all cell lines (11-, 9-, 16- and 128-fold in 22Rv1, LNCaP, PC-3 and PNT1A in the highest zinc(II) concentrations). Other miRNAs demonstrate cell line-specific trends. Expressions of, miRNA 320 in PC3 (up to 2.2-fold, $\mathrm{r}=0.96$ at $\mathrm{p}=0.038$ ) and miRNA 375 in PNT1A cell line (up to 38 -fold, $r=0.98$ at $p=0.014$ ) were significantly elevated after zinc(II) addition. In addition, miRNA 296-3p showed subtle (1.4-fold), but highly significant increase $(r=0.98$ at $p=0.005)$ in 


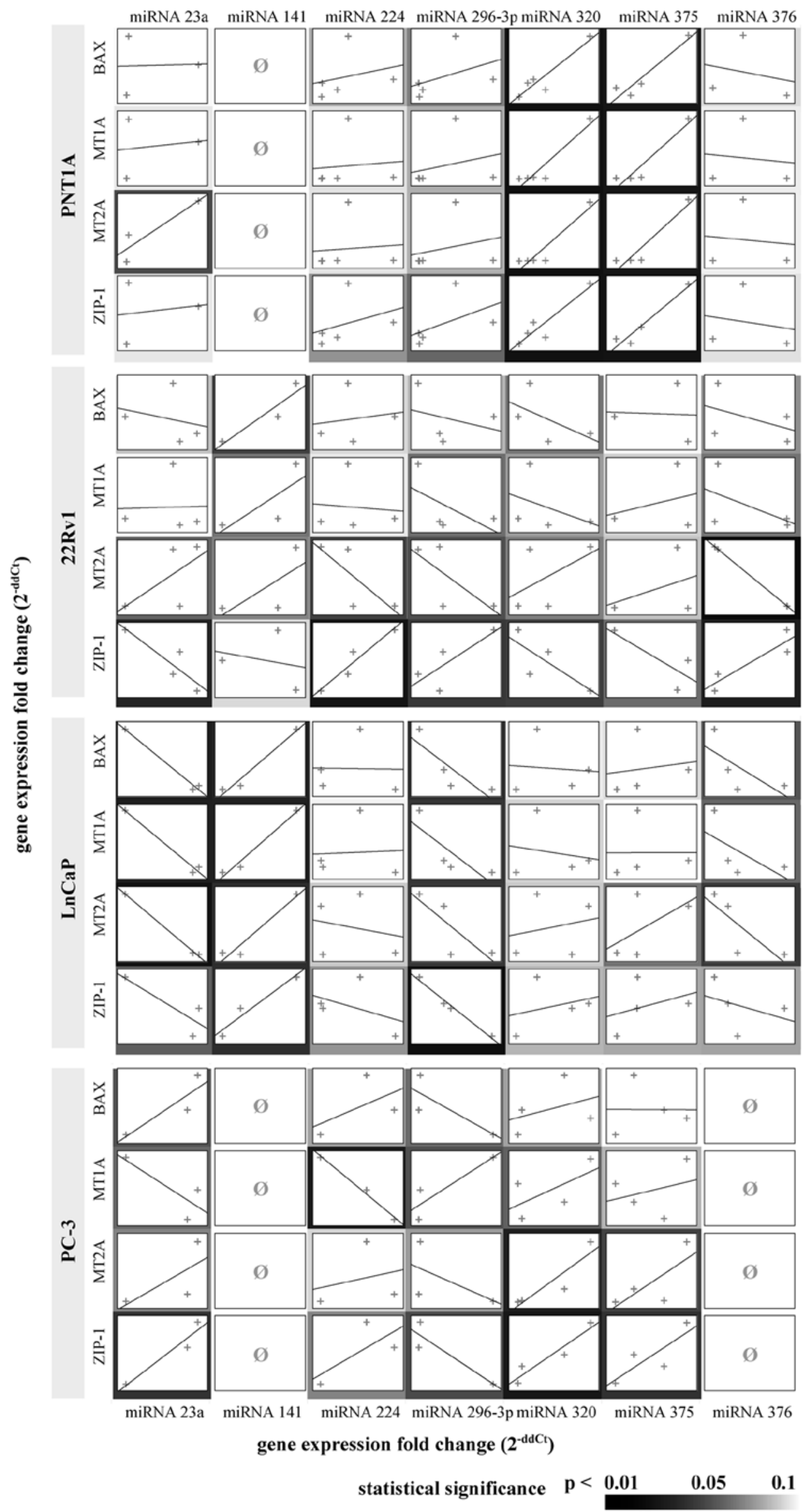

Figure 5. Correlation between miRNA and mRNA expression levels. The shade of the boxes shows the significance of the correlation. The darker the bracket is, the more significant is the correlation. $\varnothing$, undetectable. 
LNCaP. Expression of miRNA 224 in 22Rv1 cell line was negatively correlated with zinc(II) concentration (7-fold decrease, $\mathrm{r}=-0.98$ at $\mathrm{p}=0.015$ ). Change in other miRNAs' levels was also observed, however, below the level of statistical significance.

Results of ZIP-1, BAX, MT2A and MTIA gene expressions after zinc(II) treatment are shown in Fig. 4. Level of $M T 2 A$ expression in PC 3 and $22 \mathrm{Rv} 1$ cell lines $(\mathrm{r}=0.86$ at $\mathrm{p}=0.05$ and $\mathrm{r}=0.93$ at $\mathrm{p}=0.021)$ and $Z I P-1$ in PNT1A $(\mathrm{r}=0.89$ at $\mathrm{p}=0.04)$ cell line were significantly increased by the zinc(II) treatment. Expression of ZIP-1 in LNCaP cell line was significantly decreased by the increasing zinc(II) concentration ( $\mathrm{r}=-0.96$ at $\mathrm{p}=0.01$ ).

To determine the relationship between miRNA 23a, 141, 224, 296-3p, 320, 375 and 376 expression profiles of our cell lines and selected mRNA expressions, correlation analysis was performed (Fig. 5). In general, we expected negative correlations between miRNA and their potential mRNA targets as the miRNAs downregulate post-transcription processes of mRNAs. In the 22Rv1 line a negative correlation between miRNA 376 and MT2A was found (r=-1.0, p=0.0001). In the PC-3 line a negative correlation between miRNA 224 and MTlA was observed $(\mathrm{r}=-0.99, \mathrm{p}=0.07)$. In LNCaP cell line, we found negative correlation between miRNA 296-3p and ZIP-1 $(\mathrm{r}=-0.99$, $\mathrm{p}=0.01$ ). No significant correlation in the PNT1A line was found. Moreover, significant positive correlation was observed between miRNA 224 and $Z I P-1$ in the 22Rvl cell line ( $\mathrm{r}=0.96, \mathrm{p}=0.04)$.

\section{Discussion}

Cancer is characterized by abnormal and uncontrolled cell division, a phenotype that arises from the misregulation of several genes. miRNAs are major regulators of gene expression, with roles in nearly every area of cell biological functions, development and survival. Therefore, it is not surprising that miRNAs are actively altered in all types of cancers (27), acting as oncogenes or tumor suppressor genes. Main criteria for the selection of all miRNAs in our study was putative binding sites in $3^{\prime}$ UTR regions in metallothionein $1 \mathrm{~A}$ and $2 \mathrm{~A}$ and thus their possible role in the metallothionein regulation.

Porkka et al found that miRNA 23a and miRNA 23b are significantly decreased in human prostate cancer in comparison with BPH (benign prostatic hyperplasia) tissue (12). Conversely, our experiments show that level of miRNA 23a in prostate cancer cell lines is increased compared with non-tumor PNT1A cell line. We assume an association between zinc(II) metabolism and miRNAs because BPH is known for its opposite zinc(II) metabolism compared to healthy tissue and prostate cancer cells. While prostate cancer cells have decreased ability to uptake zinc(II) ions, BPH accumulates zinc(II) in higher amounts compared to 'healthy' prostate cells (14,28-30). Besides the miRNA 23a, no other miRNA studied here showed similar trend in the cell lines. On the other hand Gao et al reported that the c-Myc oncogenic transcription factor transcriptionally represses miR-23a and miR-23b (31). Nevertheless, the role and function of altered miRNA 23a in prostate cancer cells and BPH remain still unknown.

The miRNA 224 is located in malignancy-associated chromosomal region. It was published that this region shows increased gene expression in some human prostate cancer cells (32). Consistent with this study we observed specific upregulation of miRNA 224 in $22 \mathrm{Rv} 1$ line compared to others. The 22Rv1 line was derived from a human prostatic carcinoma xenograft, CWR22R, a primary tumor of prostate cancer. Apart from prostate tumors, elevation of miRNA 224 was described in other tumors. Profiling miRNA expression in hepatocellular carcinoma by Wang et al revealed miRNA 224 upregulation (33). Furthermore, consistent with the microarray data, Prueitt $e t$ al found a significantly higher expression of mature miRNA 224 in PNI (perineural invasion) tumors when compared with non-PNI tumors (34). Collectively, our results suggest that miRNA 224 is a promising biological marker of aggressive metastatic forms of prostate cancer and this miRNA should undergo further investigation also on primary tumors.

All prostate tumor cell lines overexpressed miRNA 375 in our experiments. Szczyrba et al previously found that miRNA 375 was upregulated in primary prostate cancer tissue (35), while this miRNA was downregulated in head and neck squamous cell carcinoma (36), esophagus (37), as well as in hepatocellular carcinoma (38). Poor outcome and relapse were associated with elevated levels of miR 375 in gastric carcinoma (39). Likewise, miRNA 375 is overexpressed in breast carcinoma, where it induces cell proliferation via a positive feed-back-loop with the estrogen receptor- $\alpha$ (40). As the expression of the estrogen receptor- $\alpha$ and receptor $\beta$ are increased during $\mathrm{CaP}$ development (41), it is possible that overexpression of miRNA 375 enhances the progression of this tumor. Moreover, Ladeiro et al (38) reported that the reduction of miRNA 375 expression was correlated to the expression of $\beta$-catenin. Based on their results, Ladeiro et al suggest $\beta$-catenin as a positive regulator of miRNA 375 expression. Moreover, $\beta$-catenin levels have been found to be increased in prostate cancer. Thus, our results are in concondance with the previously described data as we found increased levels of miRNA 375 in all tumor cell lines.

Key objective of this study was to describe the association of miRNAs and zinc(II)-associated genes. Metallothionein expression was shown to correlate strongly with Gleason score $(\mathrm{p}<0.001)$ and significantly with pathological staging and Ki-67 immunostaining ( $\mathrm{p}<0.001, \mathrm{p}<0.05$, respectively) (42). Our previous study also provided evidence of the association between metallothionein (MT) expression and prostate tumor progression (43). In this study we found significantly increased mRNA levels of $M T 2 A$ isoform in tumor cell lines. Our previous work also suggests MT2A is a major isoform of MT in prostate $(43,44)$. Contrary to mRNA, significantly reduced level of MT protein in tumor lines was observed. Due to these facts, posttranscriptional gene silencing can be involved and different levels of miRNAs in tumor and non-tumor lines can be expected. None of the miRNA analyzed here correlated with MT mRNA level after zinc(II) treatment in the prostate cell lines. It can be assumed according to our results that miRNAs act differently in each cell line. In the 22Rv1 tumor line a negative correlation between miRNA 376 and MT2A isoform was found. In the PC-3 line a negative correlation between miRNA 224 and MT1A was seen. Despite the observed negative correlations between miRNAs and MT after zinc(II) treatment, further exeriments to prove these findings in tissues and patient samples are required.

In terms of other genes involved in zinc(II) metabolism, the baseline expression of Bax mRNA was significantly higher in LNCaP cell line in comparison with non-tumor PNT1A. High 
Bax protein expression was shown by Amirghofran et al in prostate cancer tissue samples (45). They found a significant correlation between Bax expression and stage of carcinoma, but not with the apoptosis index, suggesting the presence of a non-functional Bax protein or the role of other proapoptotic molecules. Despite of increased level of Bax, we did not observe the enhanced apoptosis in LNCaP cell line compared to other cell lines, which supports the results of Amirghofran et al (45).

We observed higher relative expression of ZIP-1 mRNA in 22Rv1 and lower relative expression of $Z I P-1$ in PC-3 cell line compared to non-tumor PNT1A. These findings are not in conflict with the published data. Franklin et al (46) and others showed that $Z I P-1$ gene expression is downregulated and zinc(II) is depleted in adenocarcinomatous glands since early stages of cancer. Nevertheless, prostate cancer cell lines still retain the ability to express ZIP-1 gene due to its downregulation by RREB zinc finger and not fatal mutations $(47,48)$. These authors did not preclude other regulatory pathways affecting ZIP-1 expression. However, we discovered strong positive correlation between miRNA 224 and ZIP-1 expression in 22Rv1. miRNA 224 expression was significantly elevated in 22Rv1. In light of these findings, we speculate that miRNA 224 serves as another potential regulator of ZIP-1 expression. Nevertheless, no 3'UTR putative binding site of ZIP-1 mRNA for any of miRNAs studied was identified using Targetscan software (http://www.targetscan. org). Thus, this correlation can either be considered as a coincidence or miRNA 224 can affect ZIP-1 expression through other, yet unidentified mediators. These fragmentary results suggest that the area deserves further research.

Nevertheless, miRNA 375 and 23a expression levels strongly suggest their potential to contribute to the diagnosis of prostate cancer and miRNA 224 might be suitable for classification of primary tumors. Since it is also possible to assess miRNA expression patterns in urine (49) or blood serum (50), these miRNAs have the potential of being valuable biomarkers for the diagnosis and prognosis of prostate cancer. An advantage of miRNA analysis is that their molecular features reduce their susceptibility to degradate in archived tissue and samples and allows application of miRNA profiling both on diagnosis and prognosis. Although computer analysis provides data regarding miRNA to metallothionein 3'UTR, the experiment results are ambiguous, pointing to cell line-specific associations between MT and selected miRNAs. Despite the huge progress in miRNA research, we are still far away from a clear genetic demonstration of the involvement of a specific miRNA in the occurrence or progression of a specific tumor.

\section{Acknowledgements}

Financial support from NanoBioTECell GA ČR P102/11/1068, doc Ceitec.02/2012 (JG), CEITEC CZ.1.05/1.1.00/02.0068 and project for conceptual development of research organization 00064203 is highly acknowledged.

\section{References}

1. Haas GP, Delongchamps N, Brawley OW, Wang CY and de La Roza G: The worldwide epidemiology of prostate cancer: perspectives from autopsy studies. Can J Urol 15: 3866-3871, 2008 .
2. Madu $\mathrm{CO}$ and $\mathrm{Lu} \mathrm{Y}$ : Novel diagnostic biomarkers for prostate cancer. J Cancer 1: 150-177, 2010.

3. Folini M, Gandellini P, Longoni N, et al: miR-21: an oncomir on strike in prostate cancer. Mol Cancer 9: 12, 2010.

4. Kojima K, Fujita Y, Nozawa Y, Deguchi T and Ito M: MiR-34a attenuates paclitaxel-resistance of hormone-refractory prostate cancer PC3 cells through direct and indirect mechanisms. Prostate 70: 1501-1512, 2010.

5. Noonan EJ, Place RF, Basak S, Pookot D and Li LC: miR-449a causes $\mathrm{Rb}$-dependent cell cycle arrest and senescence in prostate cancer cells. Oncotarget 1: 349-358, 2010.

6. Brase JC, Johannes M, Schlomm T, et al: Circulating miRNAs are correlated with tumor progression in prostate cancer. Int J Cancer 128: 608-616, 2011.

7. Ozen M, Creighton CJ, Ozdemir M and Ittmann M: Widespread deregulation of microRNA expression in human prostate cancer. Oncogene 27: 1788-1793, 2008.

8. Cronauer MV, Schulz WA, Burchardt T, et al: The androgen receptor in hormone-refractory prostate cancer: relevance of different mechanisms of androgen receptor signaling (Review). Int J Oncol 23: 1095-1102, 2003.

9. He L and Hannon GJ: MicroRNAs: small RNAs with a big role in gene regulation. Nat Rev Genet 5: 522-531, 2004.

10. Fendler A, Jung M, Stephan C, et al: miRNAs can predict prostate cancer biochemical relapse and are involved in tumor progression. Int J Oncol 39: 1183-1192, 2011.

11. Gaur A, Jewell DA, Liang Y, et al: Characterization of microRNA expression levels and their biological correlates in human cancer cell lines. Cancer Res 67: 2456-2468, 2007.

12. Porkka KP, Pfeiffer MJ, Waltering KK, Vessella RL, Tammela TLJ and Visakorpi T: MicroRNA expression profiling in prostate cancer. Cancer Res 67: 6130-6135, 2007.

13. Kelly BD, Miller N, Healy NA, Walsh K and Kerin MJ: A review of expression profiling of circulating microRNAs in men with prostate cancer. BJU Int: May 22, 2012 (Epub ahead of print).

14. Costello LC and Franklin RB: Zinc is decreased in prostate cancer: an established relationship of prostate cancer! J Biol Inorg Chem 16: 3-8, 2011.

15. Gumulec J, Masarik M, Krizkova S, et al: Insight to physiology and pathology of zinc(II) ions and their actions in breast and prostate carcinoma. Curr Med Chem 18: 5041-5051, 2011.

16. Beyersmann D and Haase H: Functions of zinc in signaling, proliferation and differentiation of mammalian cells. Biometals 14: 331-341, 2001.

17. Haase I, Evans R, Pofahl R and Watt FM: Regulation of keratinocyte shape, migration and wound epithelialization by IGF-1- and EGF-dependent signalling pathways. J Cell Sci 116: 3227-3238, 2003.

18. Samet JM, Dewar BJ, Wu WD and Graves LM: Mechanisms of $\mathrm{Zn}(2+)$-induced signal initiation through the epidermal growth factor receptor. Toxicol Appl Pharmacol 191: 86-93, 2003.

19. Percival MD, Yeh B and Falgueyret JP: Zinc dependent activation of cAMP-specific phosphodiesterase (PDE4A). Biochem Biophys Res Commun 241: 175-180, 1997.

20. Hogstrand C, Kille P, Nicholson RI and Taylor KM: Zinc transporters and cancer: a potential role for ZIP7 as a hub for tyrosine kinase activation. Trends Mol Med 15: 101-111, 2009.

21. Yamasaki S, Sakata-Sogawa K, Hasegawa A, et al: Zinc is a novel intracellular second messenger. J Cell Biol 177: 637-645, 2007.

22. Feng P, Li TL, Guan ZX, Franklin RB and Costello LC: The involvement of Bax in zinc-induced mitochondrial apoptogenesis in malignant prostate cells. Mol Cancer 7: 25, 2008.

23. Huynh $\mathrm{H}$ : Induction of apoptosis in rat ventral prostate by finasteride is associated with alteration in MAP kinase pathways and Bcl-2 related family of proteins. Int J Oncol 20: 1297-1303, 2002.

24. Eckschlager T, Adam V, Hrabeta J, Figova K and Kizek R: Metallothioneins and cancer. Curr Protein Pept Sci 10: 360-375, 2009.

25. Eide DJ: Zinc transporters and the cellular trafficking of zinc. Biochim Biophys Acta 1763: 711-722, 2006.

26. Sztalmachova M, Hlavna M, Gumulec J, et al: Effect of zinc(II) ions on the expression of pro- and anti-apoptotic factors in high-grade prostate carcinoma cells. Oncol Rep 28: 806-814, 2012 .

27. Croce CM: Causes and consequences of microRNA dysregulation in cancer. Cell Oncol 32: 161-162, 2010.

28. Bataineh ZM, Hani IHB and Al-Alami JR: Zinc in normal and pathological human prostate gland. Saudi Med J 23: 218-220, 2002 . 
29. Goel T and Sankhwar SN: Comparative study of zinc levels in benign and malignant lesions of the prostate. Scand J Urol Nephrol 40: 108-112, 2006.

30. Sapota A, Darago A, Taczalski J and Kilanowicz A: Disturbed homeostasis of zinc and other essential elements in the prostate gland dependent on the character of pathological lesions. Biometals 22: 1041-1049, 2009.

31. Gao P, Tchernyshyov I, Chang TC, et al: c-Myc suppression of $\mathrm{miR}-23 \mathrm{a} / \mathrm{b}$ enhances mitochondrial glutaminase expression and glutamine metabolism. Nature 458: 762-765, 2009.

32. Glinsky GV, Krones-Herzig A and Glinskii AB: Malignancyassociated regions of transcriptional activation: Gene expression profiling identifies common chromosomal regions of a recurrent transcriptional activation in human prostate breast, ovarian, and colon cancers. Neoplasia 5: 218-228, 2003.

33. Wang Y, Lee ATC, Ma JZI, et al: Profiling microRNA expression in hepatocellular carcinoma reveals microRNA-224 up-regulation and apoptosis inhibitor-5 as a microRNA-224-specific target. J Biol Chem 283: 13205-13215, 2008

34. Prueitt RL, Yi M, Hudson RS, et al: Expression of microRNAs and protein-coding genes associated with perineural invasion in prostate cancer. Prostate 68: 1152-1164, 2008.

35. Szczyrba J, Nolte E, Wach S, et al: Downregulation of Sec23A protein by miRNA-375 in prostate carcinoma. Mol Cancer Res 9: 791-800, 2011.

36. Tsukamoto Y, Nakada C, Noguchi T, et al: MicroRNA-375 is downregulated in gastric carcinomas and regulates cell survival by targeting PDK1 and 14-3-3 zeta. Cancer Res 70: 2339-2349, 2010.

37. Mathe EA, Nguyen GH, Bowman ED, et al: microRNA expression in squamous cell carcinoma and adenocarcinoma of the esophagus: associations with survival. Clin Cancer Res 15 6192-6200, 2009.

38. Ladeiro Y, Couchy G, Balabaud C, et al: microRNA profiling in hepatocellular tumors is associated with clinical features and oncogene/tumor suppressor gene mutations. Hepatology 47: 1955-1963, 2008.

39. Zhang X, Yan Z, Zhang J, et al: Combination of hsa-miR-375 and hsa-miR-142-5p as a predictor for recurrence risk in gastric cancer patients following surgical resection. Annal Oncol 22 2257-2266, 2011.
40. Simonini PDR, Breiling A, Gupta N, et al: Epigenetically deregulated microRNA-375 is involved in a positive feedback loop with estrogen receptor alpha in breast cancer cells. Cancer Res 70: 9175-9184, 2010

41. Ito T, Tachibana M, Yamamoto S, Nakashima J and Murai M: Expression of estrogen receptor (ER-alpha and ER-beta) mRNA in human prostate cancer. Eur Urol 40: 557-563, 2001.

42. Athanassiadou P, Bantis A, Gonidi M, et al: The expression of metallothioneins on imprint smears of prostate carcinoma: correlation with clinicopathologic parameters and tumor proliferative capacity. Tumori 93: 189-194, 2007.

43. Gumulec J, Masarik M, Krizkova S, et al: Evaluation of alpha-methylacyl-CoA racemase, metallothionein and prostate specific antigen as prostate cancer prognostic markers. Neoplasma 59: 191-200, 2012.

44. Masarik M, Gumulec J, Hlavna M, et al: Monitoring of the prostate tumour cells redox state and real-time proliferation by novel biophysical techniques and fluorescent staining. Integr Biol 4: 672-684, 2012.

45. Amirghofran Z, Monabati A and Gholijani N: Apoptosis in prostate cancer: bax correlation with stage. Int J Urol 12: 340-345, 2005.

46. Franklin RB, Feng P, Milon B, et al: hZIP1 zinc uptake transporter down regulation and zinc depletion in prostate cancer. Mol Cancer 4: 32, 2005.

47. Thiagalingam A, DeBustros A, Borges M, et al: RREB-1, a novel zinc finger protein, is involved in the differentiation response to Ras in human medullary thyroid carcinomas. Mol Cell Biol 16: 5335-5345, 1996.

48. Zou J, Milon BC, Desouki MM, Costello LC and Franklin RB: hZIP1 zinc transporter down-regulation in prostate cancer involves the overexpression of Ras responsive element binding protein-1 (RREB-1). Prostate 71: 1518-1524, 2011.

49. Hanke M, Hoefig K, Merz H, et al: A robust methodology to study urine microRNA as tumor marker: microRNA-126 and microRNA-182 are related to urinary bladder cancer. Urol Oncol 28: 655-661, 2010.

50. Mitchell PS, Parkin RK, Kroh EM, et al: Circulating microRNAs as stable blood-based markers for cancer detection. Proc Natl Acad Sci USA 105: 10513-10518, 2008. 